\title{
UBIQUITER CIRCOVIRUS SEQUENCES RAISE CHALLENGES IN LABORATORY DIAGNOSIS: THE CASE OF HONEY BEE AND BEE MITE, REPTILES, AND FREE LIVING AMOEBAE
}

\author{
SZILVIA MARTON ${ }^{1}$, KATALIN IHÁSZ ${ }^{1}$, GYÖRGY LENGYEL ${ }^{2}$, \\ SZILVIA L. FARKAS ${ }^{1}$, ÁDÁM DÁN ${ }^{3}$, \\ PETRA PAULUS ${ }^{3}$, KRISZTIÁN BÁNYAi ${ }^{1}$ and ENIKÖ FEHÉR ${ }^{1 *}$ \\ ${ }^{1}$ Institute for Veterinary Medical Research, Centre for Agricultural Research, Hungarian \\ Academy of Sciences, Budapest, Hungary \\ ${ }^{2}$ Military Medical Centre, Hungarian Defence Forces, Budapest, Hungary \\ ${ }^{3}$ Veterinary Diagnostic Directorate, National Food Chain Safety Office, Budapest, Hungary
}

(Received: 20 November 2014; accepted: 10 January 2015)

Circoviruses of pigs and birds are established pathogens, however, the exact role of other, recently described circoviruses and circovirus-like viruses remains to be elucidated. The aim of this study was the detection of circoviruses in neglected host species, including honey bees, exotic reptiles and free-living amoebae by widely used broad-spectrum polymerase chain reaction (PCR) assays specific for the replication initiation protein coding gene of these viruses. The majority of sequences obtained from honey bees were highly similar to canine and porcine circoviruses, or, were distantly related to dragonfly cycloviruses. Other rep sequences detected in some honey bees, reptiles and amoebae showed similarities to various rep sequences deposited in the GenBank. Back-to-back PCR primers designed for the amplification of whole viral genomes failed to work that suggested the existence of integrated rep-like elements in many samples. Rolling circle amplification and exonuclease treatment confirmed the absence of small circular DNA genomes in the specimens analysed. In case of honey bees Varroa mite DNA contamination might be a source of the identified endogenous rep-like elements. The reptile and amoebae rep-like sequences were nearly identical with each other and with sequences detected in chimpanzee feces raising the possibility that detection of novel or unusual rep-like elements in some host species might originate from the microbial community of the host. Our results indicate that attention is needed when broad-spectrum rep gene specific polymerase chain reaction is chosen for laboratory diagnosis of circovirus infections.

Keywords: circovirus, integrated, rep, sequencing

* Corresponding author; E-mail: feher.eniko@agrar.mta.hu 


\section{Introduction}

The amount of sequence information about viruses with single-stranded circular DNA genome increased sharply over the past few years in line with the development of high-throughput sequencing techniques that are widely used in virological investigations of eukaryotic organisms and environmental samples $[1,2]$. Much has been learnt about the veterinary importance of some members of the Circoviridae family (including Porcine circovirus-2 and Beak and feather disease virus within the Circovirus genus and Chicken anaemia virus within the Gyrovirus genus), affecting worldwide a handful of animal host species, including pigs and poultry [3-6]. Recently, novel circoviruses (CV) and cycloviruses $(\mathrm{CyV})$ have been described in various tissues and excretes (e.g., stool, skin, cerebrospinal fluid, blood and nasopharyngeal aspirate samples) of mammals (e.g., beef, goat, camel, sheep, chimpanzee, dog, mink, bat and humans) [7-14], birds (e.g., swan, pigeon, gull, duck, goose, raven, canary, finch, and starling) [5, 7, 10, 15-22], fish (e.g., barbel, European catfish, European eel) [23-25], and insects (e.g., dragonfly and cockroach) [7, 26, 27]. Metagenomic analyses have revealed that circovirus-like viruses with a different genome length and structure are also prevalent in the environmental specimens (e.g., water, sewage, soil), although, the source and the origin of these viruses has remained unknown $[1,2,28]$.

CVs are amongst the smallest animal viruses with a genome length of 1700-2000 nt, which encode two main open reading frames (ORF); one ORF encodes the capsid protein and one supports the virus replication $[1,7]$. The ORF encoding the replication initiation protein is referred to as rep gene and contains well conserved sequences that are common among the corresponding circo-, gemini- and nanovirus sequences $[1,7]$. The encoded Rep protein of these viruses plays a pivotal role in the initiation and progress of viral replication [1]. Furthermore, rep-like sequences were detected not only in the genome of exogenous viruses but were also described as integrated genomic elements in plasmids, transposons, phages, bacteria, and a wide variety of eukaryotic organisms, including unicellular organisms, plants, fungi and animals [29, 30].

Recently, we have participated in the survey of viruses affecting Hungarian honey bees (Apis mellifera) that covered diagnostic testing of known bee-viruses and some other broadly distributed viruses, including CVs. Also, we investigated the role of various viruses in the deaths of exotic reptiles perished in captivity by screening viral agents in tissue samples. A third study aimed at investigating the role of free-living amoebae (FLA) in the carriage of infectious agents, including CVs. When relevant data from these independent studies were merged we ob- 
served some intriguing phenomena about CVs. Because we felt that our findings have implications of $\mathrm{CV}$ diagnostics we undertook to summarize our results in the following report.

\section{Materials and methods}

\section{Samples}

Honey bee and Varroa destructor mite samples were collected for a pilot study in Hungary. Honey bee specimens, each including eight or nine animals, were homogenized with the TissueLyser (Qiagen) high-throughput disruption instrument according to the manufacturer's recommendations. Two hundred $\mu 1$ of the supernatants were used for nucleic acid extraction by the MagNA Pure LC Total Nucleic Acid Isolation Kit on the MagNA Pure LC Instrument (Roche Diagnostics).

Reptile carcasses were sent to our laboratory in 2013 for virus diagnostics. Pooled samples of kidney, heart, lung, spleen, liver, intestine, stomach, trachea, oesophagus and tongue of five animals belonging to chelonian species (Testudines and Trachemys species), two Schneider's skink (Eumeces schneideri), one leopard gecko (Eublepharis macularius), and two ball pythons (Python regius) were used for virus detection. DNA was isolated with DNeasy Blood \& Tissue kit (Qiagen) according to the manufacturer's instructions.

FLA species were isolated from surface water samples including the river Danube, Lake Balaton, and the Lake of City Park, Budapest. Non-nutrient agar plates (1.5\% agar in Page's saline) coated with heat inactivated $\left(60{ }^{\circ} \mathrm{C}, 1\right.$ hour) Escherichia coli were used for isolation of FLA. Amoebic trophozoites were scraped from the agar surface and resuspended in PBS. DNA was extracted with DNeasy Blood \& Tissue kit (Qiagen) according to the manufacturer's instructions. The amoebae were identified by PCR specific for FLA and by sequencing of the resulting amplicons [31].

Purified DNA of CV isolates as well as clinical samples from pig (Porcine circovirus-2, PCV-2), pigeon, duck, goose and fish one each positive for CV were applied as positive control in the molecular biological analyses.

\section{Laboratory methods}

Broad-range PCR assays specific for the conservative rep region of $\mathrm{CVs}$ and $\mathrm{CyVs}$ were utilized using published oligonucleotides and protocols $[10,15]$. 
Twenty-five $\mu 1$ reaction mixtures were used for both PCR systems that contained $2.5 \mathrm{mM} \mathrm{MgCl}, 200 \mu \mathrm{M}$ dNTP mix, $400 \mathrm{nM}$ of each primer, $1 \mathrm{X}$ Taq Buffer with $\mathrm{KCl}, 1 \mathrm{U}$ Taq DNA polymerase (Thermo Fisher Scientific) and 1-3 $\mu 1$ of nucleic acid samples. The cycling protocols used for the PCR with CV-F1/CV-R1 and the nested CV-F2/CV-R 2 primers were as follows: $95^{\circ} \mathrm{C}$ for 3 min denaturation, 40 cycles of $95{ }^{\circ} \mathrm{C}$ for $30 \mathrm{~s}, 52^{\circ} \mathrm{C}$ (first round of the PCR) or $56^{\circ} \mathrm{C}$ (second round of PCR) for $30 \mathrm{~s}$ and $72{ }^{\circ} \mathrm{C}$ extension for $1 \mathrm{~min}$, and a final extension step at $72{ }^{\circ} \mathrm{C}$ for $10 \mathrm{~min}$ [10]. The cycling protocols used for the PCR with $\mathrm{Cv}-\mathrm{s} / \mathrm{Cv}$-as and the nested $\mathrm{Cn}-\mathrm{s} / \mathrm{Cv}$-as primers were as follows: $95^{\circ} \mathrm{C}$ for 3 min denaturation, 45 cycles of $95^{\circ} \mathrm{C}$ for $30 \mathrm{~s}, 46{ }^{\circ} \mathrm{C}$ (first round of PCR) or $56^{\circ} \mathrm{C}$ (second round of PCR) for $1 \mathrm{~min}$ and $72{ }^{\circ} \mathrm{C}$ extension for $1 \mathrm{~min}$, and a final extension step at $72{ }^{\circ} \mathrm{C}$ for $10 \mathrm{~min}$ [15].

PCR products were purified by the Geneaid Gel/PCR DNA Fragments Extraction kit(Geneaid Biotech) after gel electrophoresis and directly sequenced using an ABI PRISM 3100-Avant Genetic Analyzer and BigDye Terminator v1.1 Cycle Sequencing Kit (Thermo Fisher Scientific).

For the amplification of whole CV genomes, back-to-back primers were designed based on the sequences obtained by diagnostic PCR and Sanger sequencing. Back-to-back PCRs were carried out in $20 \mu \mathrm{l}$ reaction volume containing $400 \mathrm{nM}$ of each primer, $250 \mathrm{mM}$ dNTP mix, 1X Phusion Green HF Buffer and $0.2 \mathrm{U}$ Phusion DNA polymerase (Thermo Fisher Scientific) and 1-2 $\mu 1$ of nucleic acid samples. The cycling protocols were as follows: $98^{\circ} \mathrm{C}$ for $30 \mathrm{~s}$ denaturation, 45 cycles of denaturation at $98{ }^{\circ} \mathrm{C}$ for $10 \mathrm{~s}$, annealing at the melting temperature of the primers for $30 \mathrm{~s}$ and extension at $72{ }^{\circ} \mathrm{C}$ for $1 \mathrm{~min}$, and the final extension step at $72{ }^{\circ} \mathrm{C}$ for $10 \mathrm{~min}$.

Rolling circle amplification (RCA) was performed with phi29 DNA polymerase from the purified nucleic acid samples. $2.5 \mu \mathrm{l}$ of nucleic acid was denatured at $95{ }^{\circ} \mathrm{C}$ for 3 minutes and immediately placed on ice slurry. The RCA reaction mix of $25 \mu \mathrm{l}$ contained $200 \mu \mathrm{M}$ of dNTP mix, $30 \mu \mathrm{M}$ of random hexamer primer mix, 1X BSA, $1 \mathrm{X}$ reaction buffer and $2.5 \mathrm{U}$ phi29 DNA polymerase (New England Biolabs). Mixtures were incubated at $30{ }^{\circ} \mathrm{C}$ for 16 hours followed by the inactivation of the enzyme at $65{ }^{\circ} \mathrm{C}$ for 10 minutes. Results of the RCA were visualized by agarose gel electrophoresis.

Exonuclease digestion of the linear DNA molecules was achieved in a total volume of $20 \mu \mathrm{l}$ containing $5 \mathrm{U}$ of Lambda Exonuclease (New England Biolabs) and 1X Lambda Exonuclease Reaction Buffer and it was incubated at $37{ }^{\circ} \mathrm{C}$ for 1 hour and heat inactivated at $75^{\circ} \mathrm{C}$ for 10 minutes. As a second step, $5 \mathrm{U}$ of Exonuclease I (New England Biolabs) was added to the reaction mixture above and was incubated at $37{ }^{\circ} \mathrm{C}$ for 1 hour and then inactivated at $80{ }^{\circ} \mathrm{C}$ for 20 minutes. 
Various amplification and digestion products were visualized by agarose gel electrophoresis using $1 \%$ agarose gel prepared with $0.5 \mathrm{X}$ TBE buffer and GelRed Nucleic Acid Gel Stain (Biotium) and the electrophoresis occurred at $100 \mathrm{~V}$ for 30-60 min.

\section{Computer analysis}

The newly generated sequences are available in Table I. Phylogenetic analysis was performed using the MEGA 6 software (http:/www.megasoftware.net/) and phylogenetic trees were constructed by the neighbour-joining statistical method with p-distance substitution model and 500 bootstrap replicates (Fig. 1). The scale bar represents the nt substitution per site.

For comparison, rep and rep-like sequences were retrieved through blast search of the Basic Local Alignment Search Tool (National Center for Biotechnology Information, http://blast.ncbi.nlm.nih.gov/Blast.cgi).

GenBank reference sequence accession numbers were as follows: Porcine circovirus-2, AY424404; Porcine circovirus-1, AY660574; Canine circovirus, KC241983, JQ821392; Dragonfly cyclovirus 6, KC512918; Dragonfly cyclovirus 3, JX185424; TN4 cyclovirus from human stool, GQ404902; PK5006 cyclovirus from human stool, GQ404844; NG12 cyclovirus from human stool, GQ404854; Chimp162 from chimpanzee stool, GQ404883; Chimp17 from chimpanzee stool, GQ404851; Beak and feather disease virus, AF071878; Duck circovirus, DQ100076; Swan circovirus EU056310; Barbel circovirus, GU799606; Barbel circovirus isolate BaCV2, JF279961; Catfish circovirus isolate H5, JQ011377; Catfish circovirus isolate H6, JQ011378; rep-like sequence of Catla catla, KF051799; rep-like sequence of Labeo rohita, KF051798.

\section{Results}

CV-like sequences detected in honey bee samples

Putative CV rep-like sequences were detected by PCR in 30 of the 186 $(16.1 \%)$ honey bee and in six (100\%) Varroa mite samples. Eight honey bee specimens were excluded from further analysis due to the low quantity of the PCR products; 22 honey bee and all six Varroa PCR products were subjected to Sanger sequencing. Sequence ambiguities were observed for four honey bee and the six Varroa samples. Altogether, 18 honey bee sequences were determined and used for phylogenetic analyses. 
Table I. Circovirus rep-like sequences detected in honey bees, reptiles (chelonian species, Schneider's skink, ball pithon) and free-living amoebae (FLA)

$>\mathrm{hb} 6$

CGTGAACCTTAAGAAGACCAGCCGTATGGGTGCCTTGAAGGCACGGTTGGGTGGTCGCG GCCATTTTGAGCCTGCTCGGGGGGATGATTGCAGCAATAGAGATTATTGCTCAAAGGGGG GCGACATATTGATTGAATCGGGCGAAGTGTCTAAGCAAGGAAAACGTAATGACTTACATG ATGCAGTGGTGACCTTGAAGGAGACGAAGAGCCTTGCTGCGGTGGCTGCTGCTCACCCCG AGACTTACGTGAAGTTCTCGCGAGGTCTGCGAGAGCTCCTGCTTATAAGCCCGGAGATGG CCACGCCCCGGAAGTGGAAGACGGAAGTGCACGTCCTCGTTGGACCTCCGGGCTGTGGC AAAAGTCGGTACTGCCTAGAGACTGCACCGGAAGCATATTGGAAGCCCCGTGG

$>\mathrm{hb} 10$

ATTTTAAGTTCAAAACGAAGCAACGATTCAACACTGTACGGGCGATCCTTGGAGGCCGTG GACACCTTGAAGGTTCAAAAGGCTCTCCTAAGCAGAATCATGACTATTGTGCGAAGTCCG GAGATTACGAAACGGTGGGGGACCTCGTTATTGCGTCACAGTCATCTCTTGAACTGGTGT GCCAAATGCTTAAAGATGGAGTACGACTGGCTCAAGTCGCAGCCGATTACCCCTCAATTT TTGTCCGTCACCACAGAGGCCTTAGAGAGCTTAGCCTTATTCTTGGAGTCGTCAAACCCAG AGATTTTAAGACTGGTAAGTGGGCCGCTGCGCGGAGCATACCGGAATCGCTCTCGCGATT CCGGGCCAATCTTTTATTAACAGTCAGGTGGATGATTCTAAGTCTTCTGGTTCTTGTTTCA GAGGTGCTTGTGTTCTATGGAGCCTCTGGCACCGGCAAGTCTCGTCGCGCGGCAGAG

$>$ hb13

CGTGAACCTTAAGAAGACCAGCCGTATGGGTGCCTTGAAGGCACGGTTGGGTGGTCGCG GCCATTTTGAGCCTGCTCGGGGGGATGACTGCAGCAATAGAGATTATTGCTCAAAGGGGG GCGATATATTGATTGAATCGGGCGAAGTGTCTAAGCAAGGGAAACGTAATGACTTACATG ATGCAGTGGTGACCTTGAAGGAGACGAAGAGCCTTGCTGCGGTGGCTGCTGCTCACCCCG AGACCTACGTGAAGTTCTCGCGAGGTCTGCGAGAGCTCCTGCTTATAAGCCCGGAGATGG CCACGCCCCGGAAGTGGAAGACGGAAGTGCACGTCCTCGTTGGACCTCCGGGCTGTGGC AAAAGTCGGTACTGCCTAGAGACTGCACCGGAAGCATATTGGAAG

\section{$>\mathrm{hb} 15$}

TGCGCTTTAGTACCATCAAACAGCATCTCGATAACCGAATCCATATTGAGAAGGCAAATG GATCCGACGAAGACAACCAAAAATACTGTTCAAAAGCAGGCGAGTTTTTTGAAACGGGT GTGCCTCACACCCAAGGGAAGAGAAACGATTTGGCGGCCGTCGTGGATACCATATCGAGT GGAGCGGACATCTCATCAGTGGCTAGGACTCACCCCACCAGTTTTATCAAATACCATCGTG GAATCAAAGAATACATCAAAACCATCAGACCTATTCCCGTGCGAGACTTCAAAACTGAGG TCCGATATTACTACGGAGAACCAGGGACGGGCAAATCCAGAACAGCATTGGAAGAGGCA AAAGAAAGAGCCG

\footnotetext{
$>$ hb19
}

GACTATGACGCCGCGCTAAGCGCTCGCTTCGCTCGCTTATGTAATAAGTTTTATGTCAAAT ATAGGCTTTATCAACTTCAAATCCAAGCGGGAGTTTAGTGTCATCAAACAAATTATCGGA GACTCTGCTCATATCGAGCCAAGCAATGGCAACGACCAACAAAATAAAGAATATTGCTCT AAAAGCGATAACTTCTGGGAATTCGGAGTTCCGGTTACGCAGGGCCAACGTACAGACCTT GAAGCTGTTGTTGAAAGAATTAGATCCTCTGATAGAATCGAACAAGTGGTGTCCGAGTAC CCTGAGACTTATATTCGTTACCACAGGGGAATTGAGAAATTGTTTGGATATATATCTAAGC GAGCTAAAAGGGACTGGAAAACTGAAACGTTCGTCTACTATGGCGAACCTGGTAGTGGG AAGTCCAGAACCGCCTATGAAATTGCCCTCGCCAGAGAGGGAGGT 
Table I. (cont.)

\begin{abstract}
$>$ hb25
CGTGAACCTTAAGAAGACCACCCGTATGGGTGCTCTGAAGGCACGCTTGGGAGGTCGCGG CCATTTTGAGCCTGCTCGGGGGGACGACTGTAGCAATCAGGCATATTGCTCAAAGGGGGG CGACATCTTGGTTGAATCGGGAGCAGTTTCTAAGCAAGGCAAGCGTAATGACTTACATGA TGCAGTGTCGACGCTGAAGGAGACGAAGAGCCTTGCCGCGGTCGCTGCTGCTCACCCCGA GACTTACGTCAAGTTCTCGCGAGGACTGCGCGAGCTTCTGCTTATAAGCCCGGAGATGGC CACGCCCCGGAAGTGGAAGACGGAAGTGAATGTCCTCGTTGGACCCCCGGGCTGTGGCA AAAGTCGGTACTGCCTAGAGACTGCACCGGATGCATATTGGAAGCCCCGTGGGAAAT

$>\mathrm{hb} 26$

CGTGAACCTTAAGAAGACCACCCGTATGGGTGCTCTGAAGGCACGCTTGGGTGGTCGCGG CCATTTTGAGCCTGCTCGGGGGGACGACTGTAGCAATCAGGCATATTGCTCAAAGGGGGG CGACATCTTGATTGAATCGGGAGTAGTTTCTAAGCAAGGCAAGCGTAATGACTTACATGAT GCAGTGTCGACGCTGAAGGAGACGAAGAGCCTTGCCGCGGTCGCTGCTGCTCACCCCGAG ACTTACGTCAAGTTCTCGCGAGGACTGCGCGAGCTTCTGCTTATAAGCCCGGAGATGGCC ACGCCCCGGAAGTGGAAGACGGAAGTGAACGTCCTCGTTGGACCCCCGGGCTGTGGCAA AAGTCGGTACTGCCTAGAGACTGCACCGGATGCATAT
\end{abstract}

$>\mathrm{hb} 34$

TGCTAGCCGTGAATTACCAACATCATTGGACCGTTTCAAAGCACTAATTAGTGAACGTGCA CACATCGAAATTGCTAAAGCAAGTGAAGAAGAAGAAAAAAACCGTCAAAAAAGGCGGT CATTATGAGGAACACGGGCGTTTAAATTCGCACGGTCAGCGAAATGACTTATACAACGTT GTAGATACCCTTTTGAATAATCAGGACGATCCCATTGGCGCTGTGGCTGAATCGCATCCAG TTGCATTTATAAAATTTCGAAGAGGCATACGTGATCTTGCAACTACCCTTAAATTAGGAGC TAAACGAAACTATCGCACAAAATTGTGTATTGTTTATGGATACCCAGGGACCGGCAAAAG TTTTGGTGTCAGACAGATATGTGATAAACTATTTGGTGAAGAT

$>\mathrm{hb} 45$

CGTGAACCTTAAGAAGACCAGCCGTATGGGTGCCTTGAAGGCACGCTTGGGTGGTCGCGG CCATTTTGAGCCTGCTCGGGGGGATGATTGCAGCAATAGAGATTATTGCTCAAAGGGGGG CGACATATTGATTGAATCGGGCGAAGTGTCTAAGCAAGGTAAACGCAATGACTTACATGA TGCAGTGGCGACCTTGAAGGACACGAAGAGCCTTGCTGCGGTGGCTGCTGCTCACCCCGA GACTTACGTGAAGTTCTCGCGAGGTCTGCGAGAGCTCCTGCTTATAAGCCCGGAGATGGC CACGCCCCGGAAGTGGAAGACGGAAGTGCACGTCCTCGTTGGACCTCCGGGCTGTGGCA AAAGTCGGTACTGCCTAGAGACTGCACCGGATGCCTATTGGAAG

\title{
$>\mathrm{hb} 46$
}

CGTGAACCTTAAGAAGACCAGTCGTATGGGTGCCTTGAAGGCACGGTTGGGTGGTCGCGG CCATTTTGAGCCTGCTCGGGGGGATGATTGCAGCAATAGAGATTATTGCTCAAAGGGGGG CGACATATTGATTGAATCGGGCGAAGTGTCTAAGCAAGGGAAACGTAATGACTTACATGA TGCAGTGGCGACCTTGAAGGATACGAAGAGCCTTGCTGCGGTGGCTGCTGCTCACCCCGA GACTTACGTGAAGTTCTCGCGAGGTCTGCGAGAGCTCCTGCTTATAAGCCCGGAGATGGC CACGCCCCGGA

$>\mathrm{hb} 53$

CGTGAACCTCAAGAAGACCACCCGTATGGGTGCCTTGAAGTCACGCTTGGGTGGTCGCGG CCATTTTGAGCCTGCTCGGGGGGACGATTGCAGCAATAGAGATTATTGCTCAAAGGGGGG CGACATCTTGATTGAATGTGGCGAAGTGTCTAAACAAGGAAAACGCAATGACTTACATGA TGCAGTGACGACCTTGAAGGAGTCGAGAAGCCTTGCTGCCGTGGCTGCTGCTCACCCCGA GACTTACGTGAAGTTCTCGCGAGGACTGCGGGAGCTGCTGCTTATAAGCCCGGAGATGAC CACGCCCCGGAAGTGGAAGACGGAAGTGCACGTCCTCTGTGGACCTCCGGGCTGTGGCA AAAGTCGGTACTGCCTAGATACTGCACCGGATGCATATTGGAAGCCCC 
Table I. (cont.)

\begin{abstract}
$>\mathrm{hb76}$
CGTGAACCTTAAGAAGACCACCCGTATGGGTGCTCTGAAGGCACGCTTGGGTGGTCGCGG CCATTTTGAGCCTGCTCGGGGGGACGACTGTAGCAATCAGGCATATTGCTCAAAGGGGGG CGATATCTTGATTGAATCGGGAGCAGTTTCTAAGCAAGGCAAACGGAATGACTTACATGA TGCAGTGTCGACGCTGAAGGTAACCAAGAGCCTTGCCGCGGTCGCTGCTGCTCACCCCGA GACTTACGTCAAGTTCTCGCGAGGACTGCGAGAGCTCCTGCTTATAAGCCCGGAGATGGC CACGCCCCGGAAGTGGAAGACGGAAGTGAACGTCCTCGTTGGACCCCCGGGCTGTGGCA AAAGTCGGTACTGCCTAGAGACTGCACCGGATGCATATTGGAAG
\end{abstract}

\begin{abstract}
$>\mathrm{hb77}$
CGTGAACCTTAAGAAGACCACCCGTATGGGTGCTCTGAAGGCACGCTTGGGTGGTCGCGG CCATTTTGAGCCTGCTCGGGGGGACGACTGTAGCAATCAGGCATATTGCTCAAAGGGGGG CGATATCTTGATTGAATCGGGAGCAGTTTCTAAGCAAGGCAAACGGAATGACTTACATGA TGCAGTGTCGACGCTGAAGGTAACCAAGAGCCTTGCCGCGGTCGCTGCTGCTCACCCCGA GACTTACGTCAAGTTCTCGCGAGGACTGCGAGAGCTCCTGCTTATAAGCCCGGAGATGGC CACGCCCCGGAAGTGGAAGACGGAAGTGAACGTCCTCGTTGGACCCCCGGGCTGTGGCA AAAGTCGGTACTGCCTAGAGACTGCACCGGATGCATATTGGAAGCCCCGTGGGAAATGGT GGGACGGCT
\end{abstract}

\begin{abstract}
$>\mathrm{hb} 79$
TGCTAGCCGTGAATTACCAACATCATTGGACCGTTTCAAAGCACTAATTAGTGAACGTGCA CACATCGAAATTGCTAAAGCAAGTGAAGAAGAAGAAAAAAACCGTCAAAAAAGGCGGT CATTATGAGGAACACGGGCGTTTAAATTCGCACGGTCAGCGAAATGACTTATACAACGTT GTAGATACCCTTTTGAATAATCAGGACGATCCCATTGGCGCTGTGGCTGAATCGCATCCAG TTGCATTTATAAAATTTCGAAGAGGCATACGTGATCTTGCAACTACCCTTAAATTAGGAGC TAAACGAAACTATCGCACAAAATTGTGTATTGTTTATGGATACCCAGGGACCGGCAAAAG TTTTGGTGTCAGACAGATATGTGATAAACTATTTGGTGAAGATCT
\end{abstract}

$>\mathrm{hb} 85$

GCAGCTCGAGGAATACCAACGACCATAGACAGATTCAAAGCCTTAATCAGCTCTCGTGCA CATGTAGAAATAGCAAGACCAACAGAAGAACAGAATCGTGCATATTGTGTAAAAGGAGGT AATTACGAAGAATATGGAAGAATCAATTCTCAAGGACAACGAAATGACCTGCACAACGTC GTGGATACTTTACTGGATGCTACAGATGACCCAATCAACGCTGTTGCTACGAATCACCCTG TTGCCTTCATTAAATTCGGACGTGGCATCAGAGATTTGGCATGTCAGTTACGCCTGGGAAC TAAGAGAACACACAGAACCAA-ACTGT-GTATTGTATGGGGTTACCCAGGTACAGGCAAAA GCTTCGGTGTTCGTCAATGTGCCGAAAAATTGGTTGGCGAAGAGAAATATATTATAA

$>$ hb91

TGCTAGCCGTGAATTACCAACATCATTGGACCGTTTCAAAGCACTAATTAGTGAACGTGCA CACATCGAAATTGCTAAAGCAAGTGAAGAAGAAGAAAAAAACCGTCAAAAAAGGCGGT CATTATGAGGAACACGGGCGTTTAAATTCGCACGGTCAGCGAAATGACTTATACAACGTT GTAGATACCCTTTTGAATAATCAGGACGATCCCATTGGCGCTGTGGCTGAATCGCATCCAG TTGCATTTATAAAATTTCGAAGAGGCATACGTGATCTTGCAACTATCCTTAAATTAGGAGC TAAACGAAACTATCGCACAAAATTGTGTATTGTTTATGGATACCCAGGGACCGGCAAAAG TTTTGGTGTCAGACAGATATGTGATAAACTATTTGGTGAAGATCTGACATTTTATAAA

$>$ hb95

CGTGAACCTTAAGAAGACCAGCCGTATGGGTGCCTTGAAGGCACGGTTGGGTGGTCGCG GCCATTTTGAGCCTGCTCGGGGGGATGATTGCAGCAATAGAGATTATTGCTCAAAGGGGG GCGACATATTGATTGAATCGGGCGAAGTGTCTAAGCAAGGGAAACGTAATGACTTACATG ATGCAGTGGCGACCTTGAAGGAGACGAAGAGCCTTGCTGCGGTGGCTGCTGCTCACCCCG AGACTTACGTGAAGTTCTCGCGAGGTCTGCGAGAGCTCCTGCTTATAAGCCCGGAGATGG CCACGCCCCGGAAGTGGAAGACGGAAGTGCACGTCCTCGTTGGACCTCCGGGCTGTGGC AAAAGTCGGTACTGCCTAGAGACTGCACCGGATGCATATTGGAAG 
Table I. (cont.)

\begin{abstract}
$>$ hb109
GTTCGCTAATTTTGTGAAGAAGCAGACTTTTAATAAAGTGAAGTGGTATTTGGGTGCCCG CTGCCACATCGAGAAAGCGAAAGGAACAGATCAGCAGAATAAAGAATACTGCAGTAAAG AAGGCAACTTACTGATTGAGTGTGGAGCTCCTAGATCTCAGGGACAACGGAGTGACCTGT CTACTGCTGTGAGTACCTTGTTGGAGAGCGGGAGTCTGGTGACCGTTGCAGAGCAGCACC CTGTAACGTTTGTCAGAAATTTCCGCGGGCTGGCTGAACTTTTGAAAGTGAGCGGGAAA ATGCAGAAGCGTGA
\end{abstract}

$>$ FLA2

TTGGGTACACAACGCCTCCATCTAGAGGTACGTCGAGGTACATCAGTAGAAGCTATAGAGT ATTGCCAGAAAGAAGGTACCCGTATAGATGGACCATGGACAGCAGGTACGCCTTCCGAGG GTCAGGGAGAGCGAACAGATATAACCAAAGTGAAAGAGATGATAGATCGTGGAGCGTCTG AGATAGAAGTAGCAGAGGCAAGCTTCGCCCTATGGTGTGTTCACCATAGGGCTTTCTTACG GTACAAAGTACTAAAAACACCTGATAGGGATTTTCAAACTTTAGTCACTGTAGTTGTAGGT CCACCTGGGACAGGG

$>$ FLA3

TTGGGTACACAACGCCTCCATCTAGAGGTACGTCGAGGTACATCAGTAGAAGCTATAGAGT ATTGCCAGAAAGAAGGTACCCGTATAGATGGACCATGGACAGCAGGTACGCCTTCCGAGG GTCAGGGAGAGCGAACAGATATAACCAAAGTGAAAGAGATGATAGATCGTGGAGCGTCTG AGATAGAAGTAGCAGAGGCAAGCTTCGCCCTATGGTGTGTTCACCATAGGGCTTTCTTACG GTACAAAGTACTAAAAACACCTGATAGGGATTTTCAAACTTTAGTCACTGTAGTTGTAGGT CCACCTGGGACAGGGAAATCACGGTACGCTAACGAGAGAGATAAAGGGGCTTTCTGGAA GCAGCATGGTACTTGG

$>$ FLA4

TTGGGTACACAACGCCTCCATCTAGAGGTACGTCGAGGTACATCAGTAGAAGCTATAGAGT ATTGCCAGAAAGAAGGTACCCGTATAGATGGACCATGGACAGCAGGTACGCCTTCCGAGG GTCAGGGAGAGCGAACAGATATAACCAAAGTGAAAGAGATGATAGATCGTGGAGCGTCTG AGATAGAAGTAGCAGAGGCAAGCTTCGCCCTATGGTGTGTTCACCATAGGGCTTTCTTACG GTACAAAGTACTAAAAACACCTGATAGGGATTTTCAAACTTTAGTCACTGTAGTTGTAGGT CCACCTGGGACAGGGAAATCACGGTACGCTAACGAGAGAGATAAAGGGGCTTTCTGGAA GCAGCATGGTACTTGG

>FLA5

GAGGTACATCAGTAGAAGCTATAGAATATTGCCAGAAAGAAGGTACCCGTATAGATGGAC CATGGACAGCAGGTACTCCTTCCGAGGGGCAGGGAGAGCGAACAGATATAACCAAAGTG AAAGAGATGATAGATCGTGGAGCATCTGAGATAGAAGTAGCAGAGGCAAGCTTCGCCCTA TGGTGTGTTCACCATAGGGCTTTCTTACGGTACAAAGTACTAAAAACACCTGATAGGGATT TTCAAACTTTAGTCACTGTAGTTGTAGGTCCACCTGGGACAGGGAAATCACGGTACGCTAA CGAGAGAGATAAAGGGGCTTTCTGGAAGCAGCATGGTACTTGG

\title{
$>$ FLA6
}

TTGGGTACACAACGCCTCCATCTCGAGGTACGTCGAGGTACATCAGTAGAAGCTATAGAAT ATTGCCAGAAAGAAGGTACCCGTATAGATGGACCATGGACAGCAGGTACTCCTTCCGAGG GGCAGGGAGAGCGAACAGATATAACCAAAGTGAAAGAGATGATAGATCGTGGAGCATCT GAGATAGAAGTAGCAGAGGCAAGCTTCGCCCTATGGTGTGTTCACCATAGGGCTTTCTTAC GGTACAAAGTACTAAAAACACCTGATAGGGATTTTCAAACTTTAGTCACTGTAGTTGTAGG TCCACCTGGGACAGGGAAATCACGGTACGCTAACGAGAGAGATAAAGGGGCTTTCTGGA AGCAGCATGGTACTTGG 
Table I. (cont.)

\begin{abstract}
$>\mathrm{T} 1$ Chelonian CGTAGAGTTCAAGCAGAAACGGACCATGAATCAAGTGAAGACTCAATTGGGTACACAACG CCTCCATCTAGAGGTACGTCGAGGTACATCAGTAGAAGCTATAGAGTATTGCCAGAAAGAA GGTACCCGTATAGATGGACCATGGACAGCAGGTACGCCTTCCGAGGGTCAGGGAGAGCGA ACAGATATAACCAAAGTGAAAGAGATGATAGATCGTGGAGCGTCTGAGATAGAAGTAGCA GAGGCAAGCTTCGCCCTTTGGTGTGTTCACCATAGGGCTTTCTTACGGTACAAAGTACTAA AAACACCTGATAGGGATTTTCAAACTTTAGTTACTGTAGTTGTAGGTCCACCTGGGACAGG GAAATCACGGTACGCTAACGAGAGAGATAAAGGGGCT
\end{abstract}

\begin{abstract}
$>\mathrm{T} 2$ Chelonian
CGTAGAGTTCAAGCAGAAACGGACAATGAATCAAGTGAAGAATCAGTTGGGTACACAACG CCTCCATCTAGAGGTACGTCGAGGTACATCAGTAGAAGCTATAGAATATTGCCAGAAAGAA GGTACCCGTATAGATGGACCATGGACAGCAGGTACTCCTTCCGAGGGGCAGGGAGAGCGA ACAGATATAACCAAAGTGAAAGAGATGATAGATCGTGGAGCATCTGAGATAGAAGTAGCA GAGGCAAGCTTCGCCCTATGGTGTGTTCACCATAGGGCTTTCTTACGGTACAAAGTACTAA AAACACCTGATAGGGATTTTCAAACTTTAGTCACTGTAGTTGTAGGTCCACCTGGGACAGG GAAATCACGGTACGCTAACGAGAGAGATAAAGGGGCT
\end{abstract}

$>$ T4 Chelonian

CGTAGAGTTCAAGCAGAAACGGACCATGAATCAAGTGAAGACTCAATTGGGTACACAACG CCTCCATCTAGAGGTACGTCGAGGTACATCAGTAGAAGCTATAGAGTATTGCCAGAAAGAA GGTACCCGTATAGATGGACCATGGACAGCAGGTACGCCTTCCGAGGGTCAGGGAGAGCGA ACAGATATAACCAAAGTGAAAGAGATGATAGATCGTGGAGCGTCTGAGATAGAAGTAGCA GAGGCAAGCTTCGCCCTTTGGTGTGTTCACCATAGGGCTTTCTTACGGTACAAAGTACTAA AAACACCTGATAGGGATTTTCAAACTTTAGTCACTGTAGTTGTAGGTCCACCTGGGACAGG GAAATCACGGTACGCTAACGAGAGAGATAAAGGGGCT

>T5 Chelonian

AAGGTACCCGTATAGATGGACCATGGACAGCAGGTACGCCTTCCGAGGGTCAGGGAGAGC GAACAGATATAACCAAAGTGAAAGAGATGATAGATCGTGGAGCGTCTGAGATAGAAGTAG CAGAGGCAAGCTTCGCCCTTTGGTGTGTTCACCATAGGGCTTTCTTACGGTACAAAGTACT AAAAACACCTGATAGGGATTTTCAAACTTTAGTTACTGTAGTTGTAGGTCCACCTGGGACA GGGAAATCACGGTACGCTAACGAGAGAGATAAAGGGGCT

>R10_Ball_pithon
CGTAGAGTTCAAGCAGAAACGGACCATGAATCAAGTGAAGACTCAATTGGGTACACAACG
CCTCCATCTAGAGGTACGTCGAGGTACATCAGTAGAAGCTATAGAATATTGCCAGAAAGAA
GGTACCCGTATAGATGGACCATGGACAGCAGGTACGCCTTCCGAGGGTCAGGGAGAGCGA
ACAGATATAACCAAAGTAAAAGAGATGATAGATCGTGGAGCGTCTGAGATAGAAGTAGCA
GAGGCAAGCTTCGCCCTATGGTGTGTTCACCATAGGGCTTTCTTACGGTACAAAGTACTAA
AAACACCTGATAGGGATTTTCAAACTTTAGTCACTGTAGTTGTAGGTCCACCTGGGACAGG
GAAATCACGGTACGCTAACGAGAGAGACAAAGGGGCT
>R12_Skink
CGTAGAGTTCAAGCAGAAACGGACAATGAATCAAGTGAAGAATCAGTTGGGTACACAACG
CCTCCATCTCGAGGTACGTCGAGGTACATCAGTAGAAGCTATAGAATATTGCCAGAAAGAA
GGTACCCGTATAGATGGACCATGGACAGCAGGTACTCCTTCCGAGGGGCAGGGAGAGCGA
ACAGATATAACCAAAGTGAAAGAGATGATAGATCGTGGAGCATCTGAGATAGAAGTAGCA
GAGGCAAGCTTCGCCCTTTGGTGTGTTCACCATAGGGCTTTCTTACGGTACAAAGTACTAA
AAACACCTGATAGGGATTTTCAAACTTTAGTCACTGTAGTTGTAGGTCCACCTGGGACAGG
GAAATCACGGTACGCTAACGAGAGAGATAAAGGGGCT




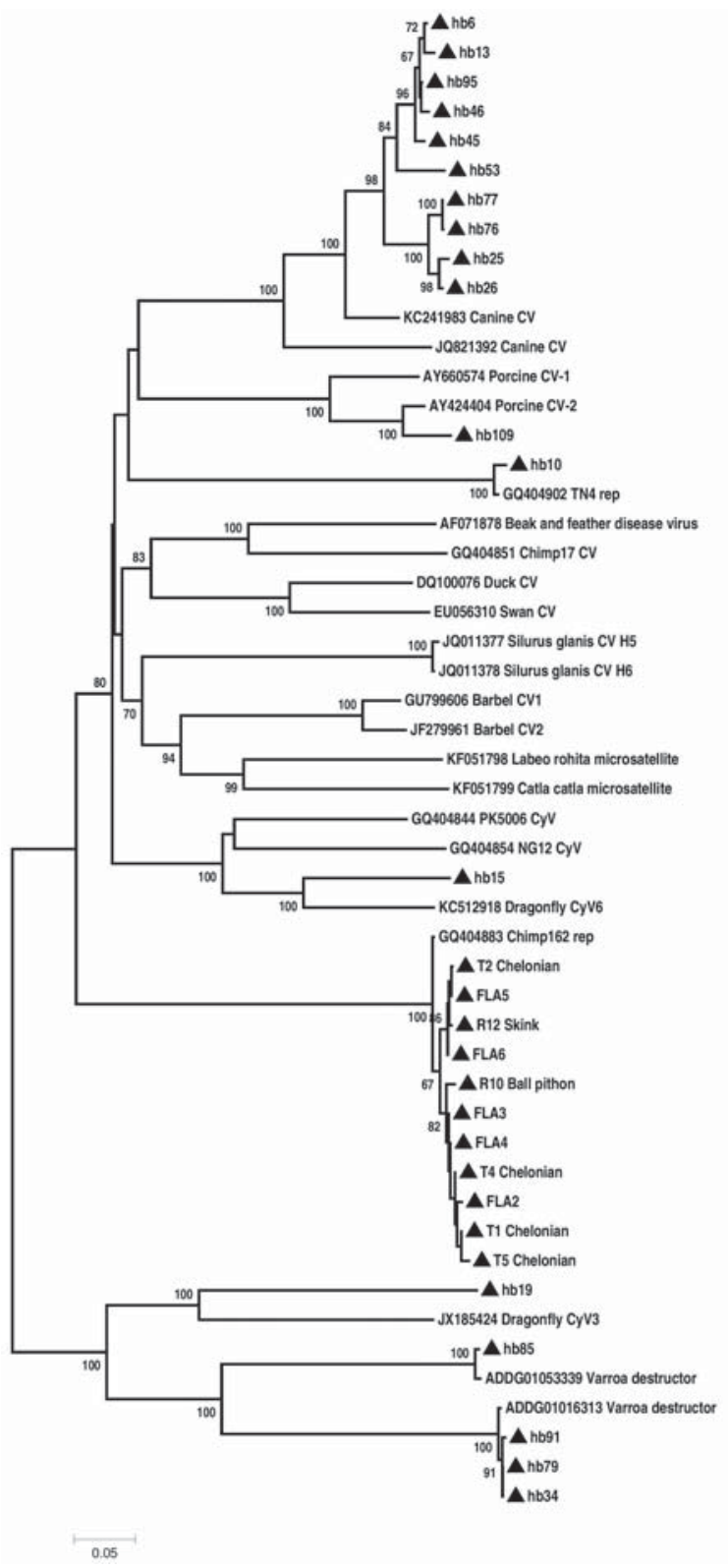

Figure 1. Phylogenetic analysis of circovirus (CV) rep-like sequences detected by PCR in honey bee (hb), Varroa destructor, reptile and free-living amoebae (FLA) samples using the neighbor-joining statistical method and p-distance substitution model of the MEGA 6 software (http://www.megasoftware.net/). The analysis based on 500 bootstrap replicates and values $>60 \%$ are represented. Hungarian sequences are labelled by triangles 
One honey bee gave amplicons with the primers designed by Halami et al. [15]. The resulting rep-like sequence (hb109) perfectly matched with PCV-2 sequences deposited in the GenBank (Fig. 1). The remaining 21 (and the eight samples that was not sequenced due to the low quantity of PCR products) honey bee and the six Varroa samples tested positive for CV exclusively with the primer set of Li et al. [10].

Ten sequences amplified from honey bees (hb6, hb13, hb25, hb26, hb45, hb46, hb53, hb76, hb77, hb95) were highly similar (90-100\% nt identity) to each other and were related (90-94\% nt identity) to the rep of a canine CV (CanCV; KC241983) (Fig. 1). The deduced aa sequences showed 93-97\% identity with this reference. Three pairs of honey bee samples (hb25 and hb26, hb45 and hb46, hb76 and hb77) originated from the same hive, but only the hb76 and hb77 nt sequences were identical. The hb10 sequence was highly similar (99\% nt and aa identity) to a partial rep-like sequence (GQ404902) from human stool sample and distantly related to rep-like sequences detected in sewage ( $<41 \% \mathrm{nt}$ identity).

The hb15 and hb19 sequences showed $83 \%$ and $63 \%$ nt identity with to the rep of dragonfly cyclovirus (DfCyV) 6 (KC512918) and DfCyV3 (JX185424), respectively. The aa identity was $80 \%$ between the hb15 and DfCyV6; the hb19 rep-like sequence contained internal stop codons.

The sequences generated from the PCR amplicons of hb34, hb79, hb85 and hb91 samples were distantly related (43-45\% nt identity) to the rep of DfCyV3, but the nt composition of the hb85 sequence greatly differed (63\% nt identity) from the nearly identical hb34, hb79 and hb91 CV-like sequences. All these divergent sequences showed $99 \%$ nt identity with respective contigs of Varroa mite whole genome shotgun sequences deposited in the GenBank (ADDG01016313, ADDG01053339). The deduced aa sequence of the hb85 rep-like sequence showed $38 \%$ aa identity with the DfCyV3 but aa sequences could not be predicted for hb34, hb39 and hb91 nt due to internal stop codons.

\section{CV-like sequences detected in reptiles}

CV rep-like sequences were detected by PCR primers of Li et al. [10] in the pooled organ samples of six reptiles $(6 / 10,60 \%)$, in particular, in four chelonian species, one skink and one ball python specimens. The primer sets of Halami et al. [15] could not amplify any CV-like sequences. The six sequences showed $98-100 \%$ nt similarity to each other without any significant differences regarding the host species. The closest relative of the sequences was a CV rep-like sequence (GQ404854) detected in chimpanzee stool; other CV rep or rep-like sequences 
were only distantly related ( $<45 \%$ nt identity) to the reptile origin rep-like sequences. The deduced aa sequences of the reptile sequences showed $99-100 \%$ similarity among each other and with the reference sequence as well.

\section{CV-like sequences detected in free-living amoebae}

Platyamoeba and Acanthamoeba species were isolated from environmental samples. rep-like sequences were detected in all of the six amoebae isolates by using the primer set of $\mathrm{Li}$ et al. [10]. The FLA-origin CV-like sequences were highly similar to each other (98-100\%), to the rep-like sequences of the reptile samples $(98-100 \%)$ and to the rep-like sequence detected in chimpanzee stool (98-100\%), and showed low similarity ( $<45 \%$ nt identity) to other CV rep or replike sequences. One amoebae rep-like sequence was too short and was not suitable for inclusion in phylogenetic analysis. The deduced aa sequences of the FLA-origin rep-like sequences showed $100 \%$ similarity with each other, with the reptile and the reference aa sequences.

\section{Results of the back-to-back PCRs, RCA and exonuclease digestion}

Fourteen back-to-back primer sets were designed on the basis of the sequencing results of the diagnostic PCRs and attempts were made to amplify the whole genomes of putative CVs in the honey bee samples. The high similarity among CV-like sequences originating from the reptiles and amoebae allowed the use of two common back-to-back primer sets for the whole genome PCRs. However, none of our attempts with the specific back-to-back PCRs were successful and RCA assays have also failed.

The efficacy of the exonuclease treatment using the Lambda Exonuclease alone or in combination with the Exonuclease I was verified with the diagnostic primer sets $[10,15]$. PCV-2, fish, pigeon, duck and goose CV positive samples were used as positive control samples in the experiment. The honey bee, reptile and amoebae DNA specimens treated with the exonucleases tested negative for the diagnostic PCRs, while the samples used as positive controls remained positive for CV-specific PCR after the DNA digestion steps. This confirmed that, at least majority of, pig, fish, pigeon, duck and goose origin CVs used in the control reaction were present in circular form, and suggested that the CV-like DNA in the test samples were in linear form. 


\section{Discussion}

Some members of the Circoviridae virus family are extensively studied since the immunosuppressive effect of these viruses contribute to the development of serious syndromes causing significant economic losses [3-6]. In the present study we aimed at the detection and characterization of CVs from various hosts to broaden the knowledge about their host specificity and pathogenic potential. CV rep-specific PCR was carried out by two, widely used universal PCR primer sets $[10,15]$. The primers designed by Li et al. [10], which are highly degenerate primer pairs, amplified rep-like sequences very efficiently; 29 of the 30 honey bee (17/18 confirmed by sequencing), six mite, six reptile and six amoebae samples (47/48) tested positive by these primers, whereas the other primer set [15] detected rep sequence only in one (1/48 tested PCR positive) honey bee specimen.

In a recent review, eukaryotic genome databases were searched systematically for small circular ssDNA viral sequences [30]. The analyses revealed that CV-like sequences may be integrated into a number of animal genomes and may represent germline infections. According to this comprehensive analysis endogenous rep-like sequences clustered with known viruses but in distinct branches and deduced aa sequences often contained inframe stop codons. In our study direct sequencing of the PCR products amplified from honey bee samples gave ambiguous results. A series of sequences was related to CanCV, DfCyV6 and PCV-2 sequences and the deduced aa sequences were not interrupted by stop codons in the amplified fragment. We suspected that these sequences may have been parts of whole viral genomes; however, back-to-back PCR, RCA and exonuclease treatment implied this hypothesis was erroneous. Of interest, the hb10 honey bee sample, along with the reptile and the amoebae rep-like sequences showed high identity with corresponding GenBank reference sequences $(\geq 98 \%$ at nt and aa level), although these references did not represent whole viral genomes. Based on the PCR and sequencing results these closely related sequences were also thought to originate from exogenous viruses, but additional laboratory methods excluded this possibility.

Five honey bee rep-like sequences (hb19, hb34, hb79, hb85 and hb91) were distantly related to DfCyV3 rep gene and four of them showed near perfect sequence match to sequences obtained by Varroa whole genome shotgun sequences [30]. Liu et al. identified multiple integrated elements in the genome of Varroa mite and according to their hypothesis this may be the consequence of multiple integration events resulting in high copy number of endogenous sequences [30]. 
These integrated rep-like sequences can be fairly diverse at various regions of the Varroa genome. In our study six Varroa mite samples were tested and rep-like sequences were detected in all six samples by PCR; however, these (and some honey bee) sequences could not be analysed by direct sequencing due to sequence ambiguities probably caused by multiple different rep-like sequences in the host genome. Thus, our findings were consistent with the report by Liu et al. [30]. In addition, the hb19, hb34, hb79, and hb91 sequences contained multiple stop codons that are very typical in integrated rep-like sequences [30]. Collectively, sequence analyses, as well as, results of back-to-back PCR, RCA and exonuclease treatment strengthened the presumption that these five divergent sequences may be endogenous rep-like elements. Although, the honey bees were carefully handled and decontaminated before nucleic acid extraction, it cannot be excluded that some residual DNA on the surface of the animals resulted in contamination and false positive results when testing honey bee samples. Thus the integrated elements detected in honey bees in this study may have been originated from Varroa mites or another organism colonizing the bee or the mite.

The rep-like sequences found in reptiles and amoebae were highly similar to rep sequences detected in chimpanzee feces but the whole genomic sequence of the reference virus encoding for that is not available. Our results indicate that the rep-like sequences of the reptiles and amoebae may be integrated elements; it is conceivable that ingested amoebae or another microbe may be the source of rep-sequences in reptiles and chimpanzee. This would explain why the obtained rep-like sequences are so conserved irrespectively of the geographical region and the host species origin. Of note, integrated rep-like elements were also detected in the unicellular parasites Giardia intestinalis and Entamoeba hystolitica [30].

PCR is a simple, time-saving and cost effective method and rep is a conservative region making broad range rep-specific primers as the choice of laboratory diagnosis of CVs and CyVs. However, the presented data together with previously published results indicate that these primers successfully amplify not only exogenous CVs and CyVs, but also endogenous rep-like genomic elements of reptiles, insects, and fish, and/or the microbial community colonizing these animals [29]. Widespread presence of endogenous rep-like elements in eukaryotic organisms and the environment draws attention on the importance of careful selection of the applied diagnostic methods. Detection and sequencing of only partial genome fragments that is routine for $\mathrm{CV}$ diagnosis may result in oversight of integrated genomic rep-like elements. In light of these findings, published data about $\mathrm{CV}$ and $\mathrm{CyV}$ diversity in various host species may require reconsideration. 


\section{Acknowledgements}

This study was supported by the Momentum program of the Hungarian Academy of Sciences. The work was partly supported by the 'EPILOBEE project, 2012-2013' cofinanced by the EU Commission with the technical assistance of the EU reference laboratory. We gratefully acknowledge the excellent technical assistance of Ágnes Juhász and Ernőné Ottinger.

\section{References}

1. Rosario, K., Duffy, S., Breitbart, M.: A field guide to eukaryotic circular single-stranded DNA viruses: insights gained from metagenomics. Arch Virol 157, 1851-1871 (2012).

2. Rosario, K., Duffy, S., Breitbart, M.: Diverse circovirus-like genome architectures revealed by environmental metagenomics. J Gen Virol 90, 2418-2424 (2009).

3. Allan, G.M., McNeilly, F., Kennedy, S., Daft, B., Ellis, J.A., Haines, D.M., Meehan, B.M., Adair, B.M.: Isolation of porcine circovirus-like viruses from pigs with a wasting disease in the USA and Europe. J Vet Diagnostic Investig 10, 3-10 (1998).

4. Niagro, F.D., Forsthoefel, A.N., Lawther, R.P., Kamalanathan, L., Ritchie, B.W., Latimer, K.S., Lukert, P.D.: Beak and feather disease virus and porcine circovirus genomes: intermediates between the geminiviruses and plant circoviruses. Arch Virol 143, 17231744 (1998).

5. Todd, D.: Circoviruses: immunosuppressive threats to avian species: a review. Avian Pathol 29, 373-394 (2000).

6. Yuasa, N., Taniguchi, T., Yoshida, I.: Isolation and some characteristics of an agent inducing anemia in chicks. Avian Dis 23, 366-385 (1979).

7. Delwart, E., Li, L.: Rapidly expanding genetic diversity and host range of the Circoviridae viral family and other Rep encoding small circular ssDNA genomes. Virus Res 164, 114121 (2012).

8. Ge, X., Li, J., Peng, C., Wu, L., Yang, X., Wu, Y., Zhang, Y., Shi, Z.: Genetic diversity of novel circular ssDNA viruses in bats in China. J Gen Virol 92, 2646-2653 (2011).

9. Kapoor, A., Dubovi, E.J., Henriquez-Rivera, J.A., Lipkin, W.I.: Complete genome sequence of the first canine circovirus. J Virol 86, 7018 (2012).

10. Li, L., Kapoor, A., Slikas, B., Bamidele, O.S., Wang, C., Shaukat, S., Masroor, M.A., Wilson, M.L., Ndjango, J.-B.N., Peeters, M., Gross-Camp, N.D., Muller, M.N., Hahn, B.H., Wolfe, N.D., Triki, H., Bartkus, J., Zaidi, S.Z., Delwart, E.: Multiple diverse circoviruses infect farm animals and are commonly found in human and chimpanzee feces. J Virol 84, 1674-1682 (2010).

11. Lian, H., Liu, Y., Li, N., Wang, Y., Zhang, S., Hu, R.: Novel circovirus from mink, China. Emerg Infect Dis 20, 1547-1549 (2014).

12. Phan, T.G., Luchsinger, V., Avendaño, L.F., Deng, X., Delwart, E.: Cyclovirus in nasopharyngeal aspirates of Chilean children with respiratory infections. J Gen Virol 95, 922-927 (2014).

13. Smits, S.L., Zijlstra, E.E., van Hellemond, J.J., Schapendonk, C.M., Bodewes, R., Schürch, A.C., Haagmans, B.L., Osterhaus, A.D.M.E.: Novel cyclovirus in human cerebrospinal fluid, Malawi, 2010-2011. Emerg Infect Dis 19, 1511-1513 (2013). 
14. Tan, L.V., van Doorn, H.R., Nghia, H.D.T., Chau, T.T.H.: Identification of a new cyclovirus in cerebrospinal fluid of patients with acute central nervous system infections. MBio 4, e00231-13 (2013).

15. Halami, M.Y., Nieper, H., Müller, H., Johne, R.: Detection of a novel circovirus in mute swans (Cygnus olor) by using nested broad-spectrum PCR. Virus Res 132, 208-212 (2008).

16. Hattermann, K., Schmitt, C., Soike, D., Mankertz, A.: Cloning and sequencing of duck circovirus (DuCV). Arch Virol 148, 2471-2480 (2003).

17. Johne, R., Fernández-de-Luco, D., Höfle, U., Müller, H.: Genome of a novel circovirus of starlings, amplified by multiply primed rolling-circle amplification. J Gen Virol 87, 1189-1195 (2006).

18. Stewart, M.E., Perry, R., Raidal, S.R.: Identification of a novel circovirus in Australian ravens (Corvus coronoides) with feather disease. Avian Pathol 35, 86-92 (2006).

19. Todd, D., Scott, A.N.J., Fringuelli, E., Shivraprasad, H.L., Gavier-Widen, D., Smyth, J.A.: Molecular characterization of novel circoviruses from finch and gull. Avian Pathol 36, 75-81 (2007).

20. Todd, D., Weston, J., Ball, N.W., Borghmans, B.J., Smyth, J.A, Gelmini, L., Lavazza, A.: Nucleotide sequence-based identification of a novel circovirus of canaries. Avian Pathol 30, 321-325 (2001).

21. Todd, D., Weston, J.H., Soike, D., Smyth, J.A.: Genome sequence determinations and analyses of novel circoviruses from goose and pigeon. Virology 286, 354-362 (2001).

22. Woods, L.W., Latimer, K.S., Barr, B.C., Niagro, F.D., Campagnoli, R.P., Nordhausen, R.W., Castro, A.E.: Circovirus-like infection in a pigeon. J Vet Diagnostic Investig 5, 609-612 (1993).

23. Doszpoly, A., Tarján, Z.L., Glávits, R., Müller, T., Benkő, M.: Full genome sequence of a novel circo-like virus detected in an adult European eel Anguilla anguilla showing signs of cauliflower disease. Dis Aquat Organ 109, 107-115 (2014).

24. Lőrincz, M., Dán, A., Láng, M., Csaba, G., Tóth, A.G., Székely, C., Cságola, A., Tuboly, T.: Novel circovirus in European catfish (Silurus glanis). Arch Virol 157, 1173-1176 (2012).

25. Lorincz, M., Cságola, A., Farkas, S.L., Székely, C., Tuboly, T.: First detection and analysis of a fish circovirus. J Gen Virol 92, 1817-1821 (2011).

26. Padilla-Rodriguez, M., Rosario, K., Breitbart, M.: Novel cyclovirus discovered in the Florida woods cockroach Eurycotis floridana (Walker). Arch Virol 158, 1389-1392 (2013).

27. Rosario, K., Marinov, M., Stainton, D., Kraberger, S., Wiltshire, E.J., Collings, D.A., Walters, M., Martin, D.P., Breitbart, M., Varsani, A.: Dragonfly cyclovirus, a novel singlestranded DNA virus discovered in dragonflies (Odonata: Anisoptera). J Gen Virol 92, 1302-1308 (2011).

28. Rosario, K., Nilsson, C., Lim, Y.W., Ruan, Y., Breitbart, M.: Metagenomic analysis of viruses in reclaimed water. Environ Microbiol. 11, 2806-2820 (2009).

29. Fehér, E., Székely, C., Lőrincz, M., Cech, G., Tuboly, T., Singh, H.S., Bányai, K., Farkas, S.L.: Integrated circoviral rep-like sequences in the genome of cyprinid fish. Virus Genes 47, 374-377 (2013).

30. Liu, H., Fu, Y., Li, B., Yu, X., Xie, J., Cheng, J., Ghabrial, S.A., Li, G., Yi, X., Jiang, D.: Widespread horizontal gene transfer from circular single-stranded DNA viruses to eukaryotic genomes. BMC Evol Biol 11, 276 (2011).

31. Tsvetkova, N., Schild, M., Panaiotov, S., Kurdova-Mintcheva, R., Gottstein, B., Walochnik, J., Aspöck, H., Lucas, M.S., Müller, N.: The identification of free-living environmental isolates of amoebae from Bulgaria. Parasitol Res 92, 405-413 (2004). 